\title{
Surgical Management of Breast Cancer Treated with Neoadjuvant Therapy
}

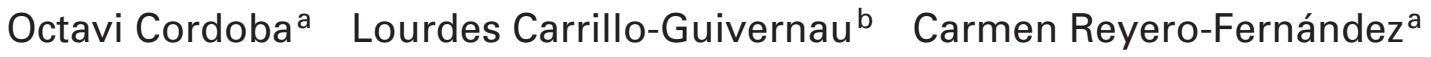 \\ ${ }^{a}$ Obstetrics and Gynecology Department, Hospital Universitari Son Espases, Palma, Spain; \\ ${ }^{b}$ Breast Cancer Unit, Obstetrics and Gynecology Department, Hospital Universitari Son Espases, Palma, Spain
}

\section{Keywords}

Breast cancer - Surgery - Neoadjuvant therapy ·

Sentinel lymph node $\cdot$ Conservative treatment

\section{Summary}

Neoadjuvant therapy (NAT) allows downstaging in some cases of breast cancer. By consequence, it may enable a more conservative surgical approach or make surgery possible in cases ineligible for surgery before NAT. In this article, we review the evidence and management recommendations for optimal surgical treatment in this setting.

(C) 2018 S. Karger GmbH, Freiburg

\section{Introduction}

Neoadjuvant therapy (NAT) in breast cancer was primarily developed to make surgery possible in locally advanced breast cancer. Later it was demonstrated that in some cases a more conservative approach may be possible after NAT [1-4]. Moreover, NAT is an excellent scenario for new treatments, as is explained by Escriváde-Romaní et al. [5] in this issue of Breast CARE. NAT includes different treatment options such as cytotoxic chemotherapy, hormonal treatment, or targeted molecular agents, depending upon the breast cancer molecular subtype (as explained by Pernaut et al. [6] in this issue of BREAST CARE).

NAT allows to start treatment of hypothetical distant disease faster than in the adjuvant setting where systemic treatment must be delayed until the end of surgical treatment and recovery. Moreover, NAT may prevent the hypothetical stimulatory effect of surgery on micrometastatic occult disease $[7,8]$. Several studies and a recent meta-analysis have shown no significant differences in over- all survival rates between NAT and adjuvant chemotherapy. The main benefit observed in patients with operable large tumors was the de-escalation of breast treatment, allowing conservative treatment in $65 \%$ of patients with NAT versus $49 \%$ in the adjuvant setting [9]. Also, as negative lymph nodes are frequently encountered in patients after NAT, de-escalation of axillary management may be possible in some cases $[2,10]$.

Surgery in the NAT setting needs careful evaluation before and after treatment. A multidisciplinary approach is needed in this scenario to achieve best results and prevent harm. Good communication between specialists during treatment is essential. This communication involves more than to discuss cases during the weekly multidisciplinary breast cancer team meetings [11]. Communication is to understand the patient's needs and all of the involved specialists' contributions. For example, the pathologist needs to understand how the radiologist evaluates treatment response and how the surgery is done in order to correctly assess the margins, and all together evaluate the need for further surgery. Only with active multidirectional communication optimal results can be achieved.

\section{Radiologic Evaluation and Tumor Marking}

Before starting NAT, the surgeon must collect and evaluate all the information that he/she needs after NAT for correct surgery planning. That involves imaging procedures for breast and axillary staging, correct assessment of multicentric or multifocal disease, and marking of the lesions and pathologic lymph nodes.

Conventional assessment includes physical examination, mammography, and ultrasound (US) of the breast and axilla. Use of digital breast tomosynthesis may improve lesion characterization compared with conventional mammography [12]. Magnetic resonance imaging (MRI) is a sensitive tool to determine the extent of the disease and is useful in the assessment of multifocal and multicentric disease [13] as this is more often underestimated on both

\section{KARGER}

() 2018 S. Karger GmbH, Freiburg

Fax +497614520714 
Table 1. EUSOMA

(2010) recommendations for magnetic resonance imaging in breast cancer staging

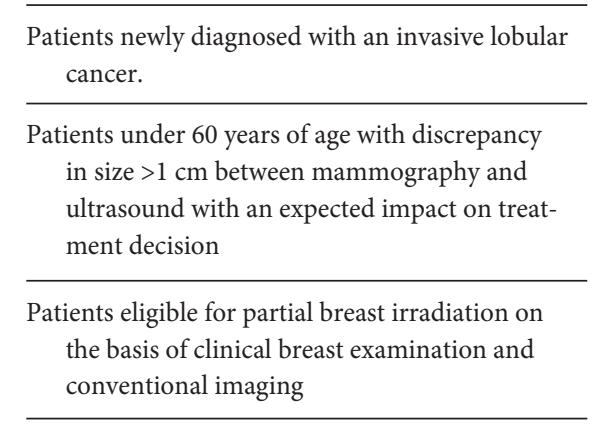

mammography and US. Moreover, MRI allows the most sensitive and accurate exploration to identify residual disease after NAT [14]. In spite of its strengths, not all patients require MRI before starting NAT. The EUSOMA recommendations for breast cancer staging with MRI continue to be useful and valid (table 1) [15]. Special care is necessary to not significantly postpone NAT initiation because of an MRI.

Additional findings in the breast of BIRADS category $>3$ have to be biopsied if the results may change the surgical treatment after NAT. Suspicious lymph nodes have to be confirmed by fine needle aspiration.

Another approach to local assessment is technetium-99m sestamibi scintimammography. This is a promising assessment tool before and after NAT and may have a role for those in whom MRI is contraindicated. However, there is insufficient data to support its routine use [16].

A single clip placed in the center of the main lesion allows good follow-up of the lesion and optimal localization at the time of surgery [17]. When a mastectomy is planned after NAT instead of the focus being on tumor response (e.g., in confirmed multicentric or inflammatory disease), clip placement is not necessary for surgery planning but may be helpful for tumor bed identification by the pathologist. The majority of microcalcifications persist after NAT even when a pathologic complete response (pCR) has been achieved; nevertheless, some microcalcifications may decrease in size or even disappear $[18,19]$. By consequence, marker placement is recommended even in patients with microcalcifications. Clip placement may also be useful in additional findings with inconclusive pathologic results, allowing resection after NAT for histological evaluation. Finally, positive axillary lymph nodes may be marked if targeted axillar dissection (TAD) might be possible after NAT.

As an alternative to metallic markers, clinically palpable tumors can be marked with a skin tattoo around the margins. This marking, however, leads to a major volume extirpation without any improvement in margins [17].

Clip marking of breast tumors is safe, and usually there is negligible displacement of the markers [20]. However, in some cases, major displacement has been reported [21]. US or mammography performed 2 weeks after clip placement allows early detection of a possible migration of the clip, and thus the necessity of a second clip placement can be evaluated.
Table 2. Indications for mastectomy regardless of response to neoadjuvant treatment
Inflammatory disease (cT4d)

Multicentric disease

Patient choice reduction risk surgery
BRCA1/BRCA2 mutation carriers wanting

All local radiologic tests should be repeated after NAT for the clinical and radiologic evaluation of response to NAT. This assessment before surgery must be done even when a radical mastectomy is planned, because imaging after NAT helps the pathologist to find residual tumor. It is recommended to perform radiologic tests 2 weeks after the last NAT cycle and within 2 weeks prior to surgery [15]. Tumor changes due to NAT (necrosis, fibrosis, fragmentation) make it difficult to assess the tumor burden with US and conventional mammography [22]. MRI is a more accurate tool to assess residual tumor burden in this setting [23]. After NAT, residual enhancement, residual tumor on US, and the markings may not coincide. For this reason, the radiologist's report must specify the response pattern and the spatial relationship between the residual tumor and the markings.

\section{Breast Surgical Treatment after NAT}

\section{Mastectomy}

There are some indications for mastectomy before NAT independent of tumor treatment response (table 2). These are inflammatory or multicentric disease, BRCA1 or BRCA2 mutations (bilateral risk reduction), and patient preference. Conservative management has been performed in patients with inflammatory disease at presentation [24]; however, information is insufficient and it should be discouraged. In patients without an initial indication for mastectomy, we must consider the new staging after NAT in the physical examination and radiologic tests. Conservative management is possible even in patients with pre-NAT involvement of the skin (cT4b) if there is good response and no skin involvement after NAT [25].

Diffuse microcalcifications after NAT constitute a controversial indication for mastectomy. According to current guidelines, all suspicious findings must be excised as persistent malignancy after NAT cannot be excluded. Recent studies have demonstrated that ductal carcinoma in situ (DCIS) may respond to NAT, achieving pCR rates of 33\%; nevertheless, microcalcifications persist in those patients [26]. Feliciano et al. [18] recently tried to identify which patients can be safely treated without excision of all microcalcifications. In their study, the authors used loss of MRI enhancement combined with mammographic findings; however, they were unable to identify a subset of patients in whom microcalcifications may be safely left in place. Kim et al. [19] identified that the correlation between microcalcifications and residual tumor burden is affected by molecular subtype. Among all breast subtypes, hormone receptor-positive/HER2-positive tumors showed the highest agreement between microcalcifications and pathology. 
There is little evidence about the safety of nipple-sparing mastectomy after NAT. Preliminary data suggest that nipple-sparing mastectomy is feasible after NAT when there is a nipple-areola complex to tumor distance of $1 \mathrm{~cm}$. Patients with good response after NAT had lower retroareolar involvement, and local relapse rates were similar to those in patients without NAT $[27,28]$.

\section{Lumpectomy}

To make a more conservative approach feasible is one of the goals of NAT. There are some special considerations with regard to breast-conserving treatment in the neoadjuvant setting. Decisions regarding breast surgical treatment should be made within the interdisciplinary team and take into account the staging post NAT, patient preferences, and contraindications to conservative treatment. Special consideration should be given to tumors with very good response such as HER2-positive tumors [25] where pCR rates of up to $66 \%$ are achieved [29-31].

Conservative surgical management after NAT is associated with an absolute increase in 15-year local recurrence of 5.5\% (95\% confidence interval 2.4-8.6; local recurrence rate $21.4 \%$ for NAT vs. $15.9 \%$ for adjuvant chemotherapy). The risk of local recurrence was significantly higher in the first 4 years in patients with NAT than in those with adjuvant treatment, with few local recurrences after year 10 [9]. There are some possible explanations which should be taken into account by the multidisciplinary team and correctly explained to the patient before starting NAT. Tumor localization during surgery is more difficult and margin interpretation may be affected by heterogeneous response patterns. Correct interpretation of radiologic tests and optimal surgical guidance, pathologic assessment, and communication between specialists are crucial for an optimal management in NAT.

Negative margins in breast tumors after NAT are defined by the same principles as in tumors without NAT [32], even in patients with invasive lobar carcinoma (ILC) [33]. Lumpectomy with negative margins is less frequently performed in ILC and in tumors without pathologic response [34].

Intraoperative US guidance to visualize residual tumor or markers allows optimal tumor excision with small amounts of breast tissue and a low percentage of second procedures needed [35]. By contrast, use of wire localization increases the volume excised without improving the need for second procedures when compared to US guidance [36]. Radioguided occult lesion localization (ROLL) and radioactive seed localization also allowed for a similar breast tissue excision volume and similar re-excision interventions when both were compared in the neoadjuvant setting [37]. US guidance requires training and experience, and to date there are no trials comparing US guidance and the ROLL technique in the neoadjuvant setting [37].

Cavity shaving allows accurate margin examination [38] and reduces the rate of positive margins and the need for second procedures $[39,40]$. This positive effect seems optimal for margin assessment in surgeries after NAT were margin status evaluation is made particularly difficult by heterogeneous response patterns. However, all published studies were performed in the adjuvant setting, and there is a lack of information on cavity shaving after NAT.
Table 3. Criteria for clinically negative axilla (cN0) previous to neoadjuvant therapy

\section{Avoiding Surgery}

Several groups have generated potential concept trials to further investigate the possibility of avoiding surgery after NAT [41, 42]. Special interest is focused on patients with HER2-positive disease where higher pCR are achieved. However, advancement in this area is limited by imaging accuracy $[14,23,43]$, and to date surgery can only be avoided in the context of a clinical trial.

\section{Surgical Lymph Node Staging}

\section{Clinically Negative Axilla (cNO) before NAT}

The criteria for a clinically negative axilla are listed in table 3 . For a long time, there was much discussion about the benefits and facts of sentinel lymph node biopsy (SLNB) before or after NAT $[44,45]$. The current recommendation of updated guidelines such as by the National Comprehensive Cancer Network (NCCN) is to perform SLNB after NAT [46] considering its accuracy with a false negative rate (FNR) of $<10 \%$ [47] and the benefits. The main benefit of performing SLNB after NAT is the avoidance of axillary lymph node dissection (ALND) in some patients due to nodal downstaging [48-50]. Moreover, axillary surgical staging after NAT has a higher accuracy in predicting locoregional recurrence [51]. Use of a dual tracer (radiolabeled colloid and blue dye) allows for a better identification rate and a reduction in the FNR in SLNB identification after NAT [52]. By contrast, SLNB before NAT should be reserved as an individualized procedure in the unusual circumstance where nodal status would provide the indication for NAT [53].

\section{Clinically Positive Axilla (cN1) before NAT}

Initial studies of SLNB after NAT in patients with $\mathrm{cN} 1$ yielded an FNR > 10\% [54, 55]; by consequence, ALND was the standard treatment in these patients. Later prospective studies provided a better characterization of technical factors and conditions that dropped the FNR below the $10 \%$ limit.

Although the FNR is not significantly different in patients with negative or positive findings on axillary US, it can identify patients with a likelihood of residual disease and higher burden of axillary disease [56]; hence, axillary US may improve the FNR according to partial data of the ACOSOG Z1071 trial [57].

The use of dual agent mapping resulted in a significantly higher sentinel lymph node identification rate and lower FNR compared to single agent mapping, highlighting the importance of technical factors in the neoadjuvant setting $[52,58,59]$. The main prospec- 
Table 4. Hospital Son Espases criteria for optimal surgical performance of surgical axillar staging after neoadjuvant treatment (NAT) in patients with a clinically positive axilla before NAT (procedure considered optimal if meeting at least 1 of those criteria)

3 sentinel lymph nodes are removed

Sentinel lymph node and marked lymph node are removed

(sentinel and marked lymph node may be the same)

Posttreatment changes are observed in the sentinel lymph node

tive studies of SLNB after NAT in cN1 patients (Z1071, SN-FNAC, SENTINA) match in that the FNR was $<10 \%$ when more than 2 lymph nodes were removed [49, 59, 60]. Marking of positive lymph nodes with radioactive seed [61-63] or US-visible markers [64] before NAT allows for TAD of the marked lymph node at the same time as SLNB. When the marked lymph node is excised at the time the SLNB is performed, the FNR is 2-6.8\% [64-66].

Use of immunohistochemical analysis to identify isolated tumor cells (ITC) on the sentinel lymph node also dropped the FNR to 8\% [49]. The presence of micrometastasis or ITC in the sentinel lymph node after NAT should be considered as a positive finding making ALND mandatory $[49,67]$. Posttreatment changes in the removed lymph nodes may be identified if previously positive lymph nodes are removed and assessed. This treatment effect is visible in $94 \%$ of previously positive lymph nodes [68].

The biological tumor subtype is associated with different axillary pCR. HER2-enriched and triple-negative tumors are especially likely to achieve axillary pCR compared to luminal breast cancer $[48,50]$. This factor should be taken into account when the whole procedure is assessed.

At our institution (Hospital Universitari Son Espases), we proceed as follows: Before starting NAT, positive lymph nodes are marked with a US-visible marker. Axillary US is performed at the end of NAT. An ALND is performed if there are no signs of radiologic response. In patients with radiologic complete response to NAT, we first perform TAD of the marked lymph nodes with intraoperative US guidance and subsequently SLNB with dual agent mapping. An X-ray test of the removed lymph nodes is performed if there is uncertainty as to the presence of the markers on the re- moved lymph nodes. Intraoperative cytological assessment of the removed lymph nodes is performed using touch test. A definitive assessment is performed with hematoxylin \& eosin and immunohistochemistry. Any positive finding in the sentinel lymph node (metastasis, micrometastasis, ITC) is considered positive, and ALND is recommended to the patient. If the sentinel lymph node is negative, all procedures are checked with Hospital Son Espases criteria (table 4). Because it is not always possible to find the clipped node or 3 sentinel lymph nodes, we also consider pathologic findings. If posttreatment changes in the lymph node are observed, we assume this to be equivalent to a marked node. If the surgical technique is considered optimal and the sentinel lymph node is negative (ypNo(sn)), no further surgery is performed and axillary radiotherapy is recommended. Patients with suboptimal technical outcome are discussed at a tumor board meeting to evaluate if ALND can be omitted or should be recommended.

\section{Axillary Lymph Node Dissection}

In cases of inflammatory breast cancer, ALND is still indicated in spite of treatment response [69]. Otherwise, any positive finding in the sentinel lymph node (metastasis, micrometastasis, ITC) is considered as positive, and ALND is recommended to the patient.

\section{Conclusion}

The surgical management of patients in the neoadjuvant setting requires a multidisciplinary approach with continuous evaluation of the procedures and results. Tailored patient management incorporates careful assessment previous to NAT, after NAT, and after surgery, and, finally, integration of all the information available.

\section{Disclosure Statement}

O. Córdoba has received fees from GE for a radioactive seeds advisory board. L. Carrillo-Guivernau and C. Reyero-Fernández certify that they have no affiliations with or involvement in any organization or entity with financial or non-financial interest in the subject matter discussed in this manuscript.

\section{References}

1 Fisher B, Bryant J, Wolmark N, et al: Effect of preoperative chemotherapy on the outcome of women with operable breast cancer. J Clin Oncol 1998;16:26722685.

2 Gianni L, Baselga J, Eiermann W, et al: Phase III trial evaluating the addition of paclitaxel to doxorubicin followed by cyclophosphamide, methotrexate, and fluorouracil, as adjuvant or primary systemic therapy: European Cooperative Trial in Operable Breast Cancer. J Clin Oncol 2009;27:2474-2481.

3 Scholl SM, Asselain B, Palangie T, et al: Neoadjuvant chemotherapy in operable breast cancer. Eur J Cancer 1991;27:1668-1671.
4 Mauriac L, MacGrogan G, Avril A, et al: Neoadjuvant chemotherapy for operable breast carcinoma larger than $3 \mathrm{~cm}$ : a unicentre randomized trial with a $124-$ month median follow-up. Ann Oncol 1999;10:47-52.

5 Escrivá-de-Romaní S, Arumí M, Zamora E, Bellet M: Neoadjuvant model as platform for research in breast cancer and novel targets under development in this field. Breast Care 2018;13:DOI: 10.1159/000492122.

6 6 Pernaut C, Lopez F, Ciruelos E: Standard neoadjuvant treatment in early/locally advanced breast cancer. Breast Care 2018;13:DOI:10.1159/000491759.

7 Fisher B, Gunduz N, Coyle J, et al: Presence of a growth-stimulating factor in serum following primary tumor removal in mice. Cancer Res 1989;49:19962001.
8 Fisher B, Saffer E, Rudock C, et al: Effect of local or systemic treatment prior to primary tumor removal on the production and response to a serum growth-stimulating factor in mice. Cancer Res 1989;49:2002-2004.

$\checkmark$ Early Breast Cancer Trialists' Collaborative Group (EBCTCG): Long-term outcomes for neoadjuvant versus adjuvant chemotherapy in early breast cancer: meta-analysis of individual patient data from ten randomised trials. Lancet Oncol 2018;19:27-39.

10 Rastogi P, Anderson SJ, Bear HD, et al: Preoperative chemotherapy: updates of national surgical adjuvant breast and bowel project protocols B-18 and B-27. J Clin Oncol 2008;26:778-785. 
11 Lamb BW, Sevdalis N, Mostafid H, et al: Quality improvement in multidisciplinary cancer teams: an investigation of teamwork and clinical decision-making and cross-validation of assessments. Ann Surg Oncol 2011; 18:3535-3543.

12 Kim WH, Chang JM, Moon H-G, et al: Comparison of the diagnostic performance of digital breast tomosynthesis and magnetic resonance imaging added to digital mammography in women with known breast cancers. Eur Radiol 2016;26:1556-1564.

13 Houssami N, Ciatto S, Macaskill P, et al: Accuracy and surgical impact of magnetic resonance imaging in breast cancer staging: systematic review and metaanalysis in detection of multifocal and multicentric cancer. J Clin Oncol 2008;26:3248-3258.

14 Marinovich ML, Houssami N, Macaskill P, et al: Metaanalysis of magnetic resonance imaging in detecting residual breast cancer after neoadjuvant therapy. J Natl Cancer Inst 2013;105:321-333.

15 Sardanelli F, Boetes C, Borisch B, et al: Magnetic resonance imaging of the breast: recommendations from the EUSOMA working group. Eur J Cancer 2010;46: 1296-1316.

16 Dialani V, Chadashvili T, Slanetz PJ: Role of imaging in neoadjuvant therapy for breast cancer. Ann Surg Oncol 2015;22:1416-1424.

17 Espinosa-Bravo M, Sao Avilés A, Esgueva A, et al: Breast conservative surgery after neoadjuvant chemotherapy in breast cancer patients: comparison of two tumor localization methods. Eur J Surg Oncol 2011;37: 1038-1043.

18 Feliciano Y, Mamtani A, Morrow M, et al: Do calcifications seen on mammography after neoadjuvant chemotherapy for breast cancer always need to be excised? Ann Surg Oncol 2017;24:1492-1498.

19 Kim Y-S, Chang JM, Moon H-G, et al: Residual mammographic microcalcifications and enhancing lesions on MRI after neoadjuvant systemic chemotherapy for locally advanced breast cancer: correlation with histopathologic residual tumor size. Ann Surg Oncol 2016; 23:1135-1142.

20 Schulz-Wendtland R, Dankerl P, Bani MR, et al: Evaluation of a marker clip system in sonographically guided core needle biopsy for breast cancer localization before and after neoadjuvant chemotherapy. Geburtshilfe Frauenheilkd 2017;77:169-175.

21 Sakamoto N, Fukuma E, Tsunoda Y, et al: Evaluation of the dislocation and long-term sonographic detectability of a hydrogel-based breast biopsy site marker. Breast Cancer 2018;DOI: 10.1007/s12282-018-0854-8.

22 Chagpar AB, Middleton LP, Sahin AA, et al: Accuracy of physical examination, ultrasonography, and mammography in predicting residual pathologic tumor size in patients treated with neoadjuvant chemotherapy. Ann Surg 2006;243:257-264.

23 Jochelson MS, Lampen-Sachar K, Gibbons G, et al: Do MRI and mammography reliably identify candidates for breast conservation after neoadjuvant chemotherapy? Ann Surg Oncol 2015;22:1490-1495.

24 Semiglazov V, Eiermann W, Zambetti M, et al: Surgery following neoadjuvant therapy in patients with HER2positive locally advanced or inflammatory breast cancer participating in the NeOAdjuvant Herceptin (NOAH) study. Eur J Surg Oncol 2011;37:856-863.

25 Debled M, Macgrogan G, Breton-Callu C, et al: Surgery following neoadjuvant chemotherapy for HER2positive locally advanced breast cancer. Time to reconsider the standard attitude. Eur J Cancer 2015;51:697704.

26 Goldberg H, Zandbank J, Kent V, et al: Chemotherapy may eradicate ductal carcinoma in situ (DCIS) but not the associated microcalcifications. Eur J Surg Oncol 2017;43:1415-1420.
27 Agresti R, Sandri M, Gennaro M, et al: Evaluation of local oncologic safety in nipple-areola complex-sparing mastectomy after primary chemotherapy: a propensity score-matched study. Clin Breast Cancer 2017; 17:219-231.

28 Santoro S, Loreti A, Cavaliere F, et al: Neoadjuvant chemotherapy is not a contraindication for nipple sparing mastectomy. Breast 2015;24:661-666.

29 Baselga J, Bradbury I, Eidtmann H, et al.; NeoALTTO Study Team: Lapatinib with trastuzumab for HER2positive early breast cancer (NeoALTTO): a randomised, open-label, multicentre, phase 3 trial. Lancet 2012;379:633-640.

30 Gianni L, Pienkowski T, Im Y-H, et al: Efficacy and safety of neoadjuvant pertuzumab and trastuzumab in women with locally advanced, inflammatory, or early HER2-positive breast cancer (NeoSphere): a randomised multicentre, open-label, phase 2 trial. Lancet Oncol 2012;13:25-32.

31 Schneeweiss A, Chia S, Hickish T, et al: Pertuzumab plus trastuzumab in combination with standard neoadjuvant anthracycline-containing and anthracyclinefree chemotherapy regimens in patients with HER2positive early breast cancer: a randomized phase II cardiac safety study (TRYPHAENA). Ann Oncol 2013; 24:2278-2284.

32 Gnant M, Harbeck N, Thomssen C: St. Gallen/Vienna 2017: a brief summary of the consensus discussion about escalation and de-escalation of primary breast cancer treatment. Breast Care (Basel) 2017;12:102107.

33 Wagner J, Boughey JC, Garrett B, et al: Margin assessment after neoadjuvant chemotherapy in invasive lobular cancer. Am J Surg 2009;198:387-391.

34 Volders JH, Haloua MH, Krekel NMA, et al: Neoadjuvant chemotherapy in breast-conserving surgery consequences on margin status and excision volumes: a nationwide pathology study. Eur J Surg Oncol 2016; 42:986-993.

35 Ramos M, Díez JC, Ramos T, et al: Intraoperative ultrasound in conservative surgery for non-palpable breast cancer after neoadjuvant chemotherapy. Int J Surg 2014;12:572-577.

36 Rubio IT, Esgueva-Colmenarejo A, Espinosa-Bravo M, et al: Intraoperative ultrasound-guided lumpectomy versus mammographic wire localization for breast cancer patients after neoadjuvant treatment. Ann Surg Oncol 2016;23:38-43.

37 Chan BKY, Wiseberg-Firtell JA, Jois RHS, et al: Localization techniques for guided surgical excision of nonpalpable breast lesions. Cochrane Database Syst Rev 2015;20:CD009206.

38 Pata G, Bartoli M, Bianchi A, et al: Additional cavity shaving at the time of breast-conserving surgery enhances accuracy of margin status examination. Ann Surg Oncol 2016;23:2802-2808.

39 Wang K, Ren Y, He J: Cavity shaving plus lumpectomy versus lumpectomy alone for patients with breast cancer undergoing breast-conserving surgery: a systematic review and meta-analysis. PLoS One 2017;12:1-14.

40 Chagpar AB, Killelea BK, Tsangaris TN, et al: A randomized, controlled trial of cavity shave margins in breast cancer. N Engl J Med 2015;373:503-510.

41 Rea D, Tomlins A, Francis A: Time to stop operating on breast cancer patients with pathological complete response? Eur J Surg Oncol 2013;39:924-930.

42 Van la Parra RFD, Kuerer HM: Selective elimination of breast cancer surgery in exceptional responders: historical perspective and current trials. Breast Cancer Res 2016;18:28.
43 Di Cosimo S, Campbell C, Azim HA, et al: The use of breast imaging for predicting response to neoadjuvant lapatinib, trastuzumab and their combination in HER2-positive breast cancer: results from NeoALTTO. Eur J Cancer 2018;89:42-48.

44 Rubio IT: Sentinel lymph node biopsy after neoadjuvant treatment in breast cancer: work in progress. Eur J Surg Oncol 2016;42:326-332.

45 Buchholz TA, Lehman CD, Harris JR, et al: Statement of the science concerning locoregional treatments after preoperative chemotherapy for breast cancer: a National Cancer Institute conference. J Clin Oncol 2008; 26:791-797.

46 Gradishar WJ, Robert CH, Anderson BO, et al: NCCN Guidelines Version 1.2018 Breast Cancer. https://www. nccn.org/professionals/physician_gls/pdf/breast_ blocks.pdf.

47 Tan VKM, Goh BKP, Fook-Chong S, et al: The feasibility and accuracy of sentinel lymph node biopsy in clinically node-negative patients after neoadjuvant chemotherapy for breast cancer - a systematic review and meta-analysis. J Surg Oncol 2011;104:97-103.

48 Boughey JC, McCall LM, Ballman KV, et al: Tumor biology correlates with rates of breastconserving surgery and pathologic complete response after neoadjuvant chemotherapy for breast cancer findings from the ACOSOG Z1071 (Alliance) prospective multicenter clinical trial. Ann Surg 2014;260:608-616.

49 Boileau J-F, Poirier B, Basik M, et al: Sentinel node biopsy after neoadjuvant chemotherapy in biopsyproven node-positive breast cancer: the SN FNAC study. J Clin Oncol 2015;33:258-264.

50 Kim JY, Park HS, Kim S, et al: Prognostic nomogram for prediction of axillary pathologic complete response after neoadjuvant chemotherapy in cytologically proven node-positive breast cancer. Medicine (Baltimore) 2015;94:e1720.

51 Mamounas EP, Anderson SJ, Dignam JJ, et al: Predictors of locoregional recurrence after neoadjuvant chemotherapy: results from combined analysis of $\mathrm{Na}-$ tional Surgical Adjuvant Breast and Bowel Project B-18 and B-27. J Clin Oncol 2012;30:3960-3966.

52 Boughey JC, Suman VJ, Mittendorf EA, et al: Factors affecting sentinel lymph node identification rate after neoadjuvant chemotherapy for breast cancer patients enrolled in ACOSOG Z1071 (Alliance). Ann Surg 2015;261:547-552.

53 Pilewskie M, Morrow M: Axillary nodal management following neoadjuvant chemotherapy: a review. JAMA Oncol 2017;3:549-555.

54 Shen J, Gilcrease MZ, Babiera GV, et al: Feasibility and accuracy of sentinel lymph node biopsy after preoperative chemotherapy in breast cancer patients with documented axillary metastases. Cancer 2007;109:12551263.

55 Alvarado R, Yi M, Le-Petross H, et al: The role for sentinel lymph node dissection after neoadjuvant chemotherapy in patients who present with node-positive breast cancer. Ann Surg Oncol 2012;19:3177-3184.

56 Boland MR, Prichard RS, Daskalova I, et al: Axillary nodal burden in primary breast cancer patients with positive pre-operative ultrasound guided fine needle aspiration cytology: management in the era of ACOSOG Z011. Eur J Surg Oncol 2015;41:559-565.

57 Boughey JC, Ballman KV, Hunt KK, et al: Axillary ultrasound after neoadjuvant chemotherapy and its impact on sentinel lymph node surgery: results from the American College of Surgeons Oncology Group Z1071 Trial (Alliance). J Clin Oncol 2015;33:3386-3393.

58 Hunt KK, Yi M, Mittendorf EA, et al: Sentinel lymph node surgery after neoadjuvant chemotherapy is accurate and reduces the need for axillary dissection in breast cancer patients. Ann Surg 2009;250:558-566. 
59 Kuehn T, Bauerfeind I, Fehm T, et al: Sentinel-lymphnode biopsy in patients with breast cancer before and after neoadjuvant chemotherapy (SENTINA): a prospective, multicentre cohort study. Lancet Oncol 2013; 14:609-618.

60 Boughey JC, Suman VJ, Mittendorf EA, et al: Sentine lymph node surgery after neoadjuvant chemotherapy in patients with node-positive breast cancer. JAMA 2013;310:1455.

61 Caudle AS, Yang WT, Mittendorf EA, et al: Selective surgical localization of axillary lymph nodes containing metastases in patients with breast cancer: a prospective feasibility trial. JAMA Surg 2015; 150:137-143.

62 Nguyen TT, Hieken TJ, Glazebrook KN, Boughey JC Localizing the clipped node in patients with node-positive breast cancer treated with neoadjuvant chemotherapy: early learning experience and challenges. Ann Surg Oncol 2017;24:3011-3016.
63 Straver ME, Loo CE, Alderliesten T, et al: Marking the axilla with radioactive iodine seeds (MARI procedure) may reduce the need for axillary dissection after neoadjuvant chemotherapy for breast cancer. Br J Surg 2010;97:1226-1231.

64 Siso C, de Torres J, Esgueva-Colmenarejo A, et al: Intraoperative ultrasound-guided excision of axillary clip in patients with node-positive breast cancer treated with neoadjuvant therapy (ILINA trial) : a new tool to guide the excision of the clipped node after neoadjuvant treatment. Ann Surg Oncol 2018;25:784-791.

65 Caudle AS, Yang WT, Krishnamurthy S, et al: Improved axillary evaluation following neoadjuvant therapy for patients with node-positive breast cancer using selective evaluation of clipped nodes: implementation of targeted axillary dissection. J Clin Oncol 2016;34: 1072-1078.
66 Boughey JC, Ballman KV, Le-Petross HT, et al: Identification and resection of clipped node decreases the false-negative rate of sentinel lymph node surgery in patients presenting with node-positive breast cancer (T0-T4, N1-N2) who receive neoadjuvant chemotherapy. Ann Surg 2016;263:802-807.

67 Rubio IT, Aznar F, Lirola J, et al: Intraoperative assessment of sentinel lymph nodes after neoadjuvant chemotherapy in patients with breast cancer. Ann Surg Oncol 2010;17:235-239.

68 Barrio AV, Mamtani A, Edelweiss M, et al: How often is treatment effect identified in axillary nodes with a pathologic complete response after neoadjuvant chemotherapy? Ann Surg Oncol 2016;23:3475-3480.

69 Reimer T, Hartmann S, Stachs A, Gerber B: Local treatment of the axilla in early breast cancer: concepts from the national surgical adjuvant breast and bowel project $\mathrm{B}-04$ to the planned intergroup sentinel mamma trial. Breast Care 2014;9:87-95. 